\section{Origin of the Solar System}

\section{Fred L. Whipple}

The Cosmogony of the Solar System. By Fred Hoyle. Pp. 168. (University College Cardiff Press/ Christopher Davies Publishers: Swansea, UK, 1979.) £7.95.

THIs book is not for the general public nor even for the serious amateur astronomer. The physics and the mathematical equations are critical to much of the discussion and, even though painstakingly explained, remain high hurdles for readers without advanced training in these subjects. On the other hand, Sir Fred writes down to research students and to his scientific peers. Had he published the book a decade or so ago as a scientific monograph with proper referencing to the host of contributors to the field, it would have been a useful summary of Sir Fred's imaginative thinking on the subject. It could have stimulated progress in understanding the complex processes that combined to form the Solar System and helped explain how we came into being. Today The Cosmogony of the Solar System serves chiefly as an historical document which can be seen to illustrate the formidable intricacy of the problem as measured in masses of data, in diverse physical processes and in time sequences of events.

Nearly everyone agrees on the gross simple picture. Collapse within a black interstellar cloud of dust and gas formed the Sun, the planets, the asteroids and the comets. Since 1950, when the comets were recognised as dirty ice balls, it has been apparent that temperature was the controlling factor in the evolution of the Solar System. The temperature decreased with increasing solar distance. Near the Sun only rocky material could freeze, so that within Jupiter's orbit dust aggregated to form planetisimals and the rocky terrestrial planets. Beyond Saturn icy cometesimals were the building blocks that formed Uranus, Neptune, probably Pluto, and some of the satellites of the great planets. Jupiter and the Sun are considered by some investigators as a double star system and in a certain sense Sir Fred agrees. This all happened 4.6 Myr ago. The details remain obscure and therein lie the controversies. Some of these complications are elaborated in The Cosmogony of the Solar System.

Sir Fred makes the basic assumption that the black cloud collapsed to the radius of Mercury's present orbit, then expanded as a rotating disk star to the limits of the planetary system. His concept fails in at least three major respects, each of which is well documented in the literature and each of which negates most of his thesis. First, a collapsing cloud in the interstellar medium cannot collapse as a simple entity. Nature really does abhor a vacuum; the collapse brought with it an influx of outlying gas and dust, horribly complicating the situation. Secondly, Sir Fred utilises "magnetic brushes" from the rapidly rotating central mass to expand the dust. $\mathrm{He}$ assumes that the expanding gas will carry along solid particles that condense as the temperature falls with distance. In fact, the gas in the disk carries an outward radial pressure that reduces the effective central gravity field. The solid particles feel only the gravity field and try to revolve faster than the gas. Thus, the gas acts as a resisting medium causing the particles to spiral inward. No rocky material, therefore, should progress beyond Jupiter's orbit, contrary to the evidence from comets and probably Uranus, Neptune and some outer satellites. Thirdly, Sir Fred adopts the "minimum mass" concept which assumes that the only matter "wasted" from the system is gas that could not condense in to the planets, asteroids and comets. The other strongest proponents of the "minimum mass" concept (to whom he does not refer) have long since abandoned the idea. Also Sir Fred's ingenious removal process-interaction with interstellar clouds-is marginally possible at best. It fails completely if nature is really as wasteful as most investigators now believe.

The accounts of the aggregation of condensates into planets are lucid and Sir Fred presents clearly the problems of explaining rotations. His thinking here follows currently conventional patterns except for Jupiter and Saturn. Great uncertainty, however, remains as to the processes which formed these giant planets. Some of the geophysical presentations will surely raise eyebrows and comments among specialists, particularly the concept that radioactive elements such as potassium, uranium and thorium were concentrated in the upper mantle of the Earth as a late time-sequence layering process rather than by magmatic differentiation. Related concepts of lunar evolution and structure are also novel and highly controversial.

As for Sir Fred's proposal that life originated in comets, I am charmed but not impressed by the picture of life forms developing in "warm little ponds," protected in their icy igloos from the cruel cold and near vacuum of open space and falling to primitive Earth at speeds exceeding eleven kilometres per second.

Fred L. Whipple is Phillips Professor of Astronomy Emeritus at Harvard University, and Director Emeritus of the Smithsonian Astrophysical Observatory, Cambridge, Massachusetts.

\section{Heroes on the climatic stage}

\section{W. S. Broecker}

Ice Ages: Solving the Mystery. By J. Imbrie and $\mathrm{K}$. Palmer Imbrie. Pp. 224. (Enslow: Short Hills, New Jersey; Macmillan: London, 1979.) $\$ 12.95 ; £ 6.95$.

ANY hit parade of prominent problems in the Earth sciences would have to include among its top ten the cause of the glaciations which have plagued the Pleistocene. Despite the prominence of this problem relatively little has been written about the history of the endeavour to explain the instability in our planet's climate. The Imbries have not only plucked this plum but have done a superb job. They provide a very readable insight into not only the development of scientific thinking on this subject but also into the person- alities of the key participants.

It is a book of heroes. Croll, from the Imbries' ancestral Scotland, receives top billing for his role in advancing the orbital theory. Milankovitch, with whose name the orbital theory is

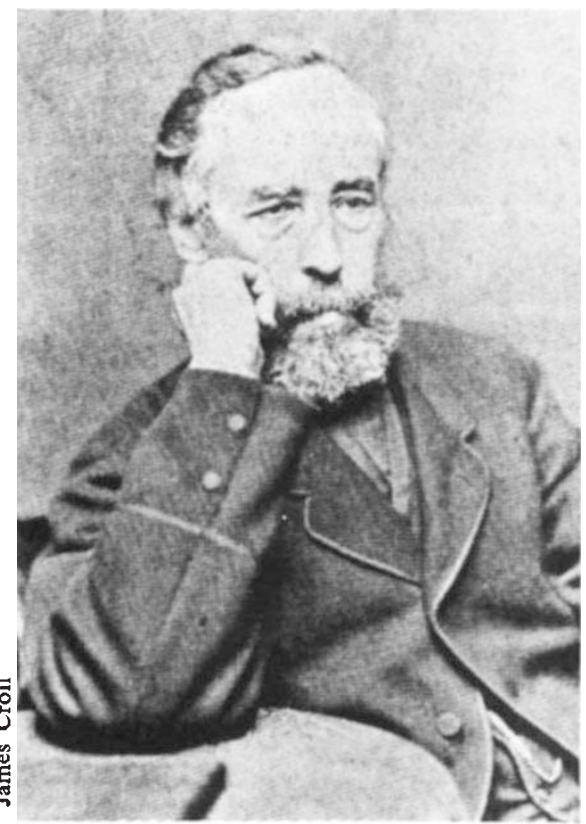

( ) Macmillan Journals Ltd 1979 
normally linked, is portrayed as a patient and confident mathematician who devotes his life to calculating the geographical and temporal variations in monthly isolation. John Imbrie's fellow detectives in the current investigation aimed at tracking down evidence in support of the orbital theory are given strong supporting roles.

The Imbries' account shows how personality conflict and serendipity complicate the path towards scientific discovery. Rarely does the key evidence emerge from a carefully planned strategy. More often it emerges from arguments between parties holding divergent views or from measurements made for some other purpose. Perhaps one of the greatest contributions of books like this is to demonstrate to the public the necessity of supporting basic research. The carefully designed pert charts of government agencies rarely produce major discoveries. Creativity cannot be programmed.

The Imbries' account shows the incredible breadth of evidence and technique which have contributed to our understanding of climatic history. The stage is successively dominated by the field geologist observing grooves and erratics, the oceanographer looking

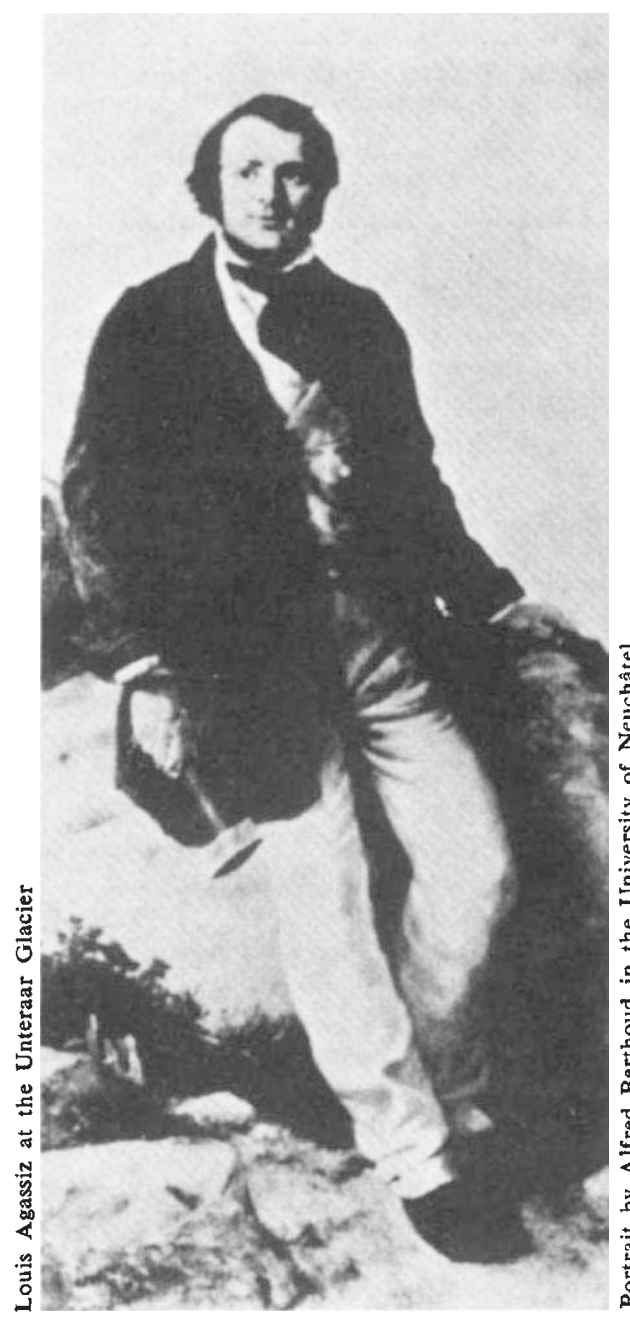

down the microscope at microfossils from deep sea cores, the isotope geochemist with his mass spectrometers and radioactivity detection systems, by the paleomagnetician, and yet again by the field geologist and by the micropaleontologist. Interwoven with field observation are mathematical calculations involving celestial mechanics, isolation dynamics, factor analyses and spectral analyses.

The Imbries are evangelists actively involved in the revival of a once prominent and then much maligned hypothesis. Although they wish the reader to believe that like continental drift $\mathscr{F}$ the Milankovitch hypothesis is being swept back into fashion by a landslide of new evidence, this reviewer suspects that the CLIMAP blitzkrieg will be slowed by a wave of resistance. Like Agassiz, Imbrie and his colleagues will encounter the Bucklands and Lyells of modern meteorology. They are steeped $\bar{\Sigma}$ in the dogma that the orbital changes are far too weak to be responsible for the awesome changes from a glacial to an interglacial world. Thus, rather than constituting the final report on solution of a major scientific problem, the Imbries' book will more likely prove to be a status report on a continuing investigation. Many new heroes will likely occupy the climatic stage before the mystery is solved.

The Imbries' enthusiasm for the isolation hypothesis in no way detracts, rather, it gives the book an excitement which allows it to transcend most treatments of the history of science. Their book does for climate what J. D. Watson's The Double Helix did for

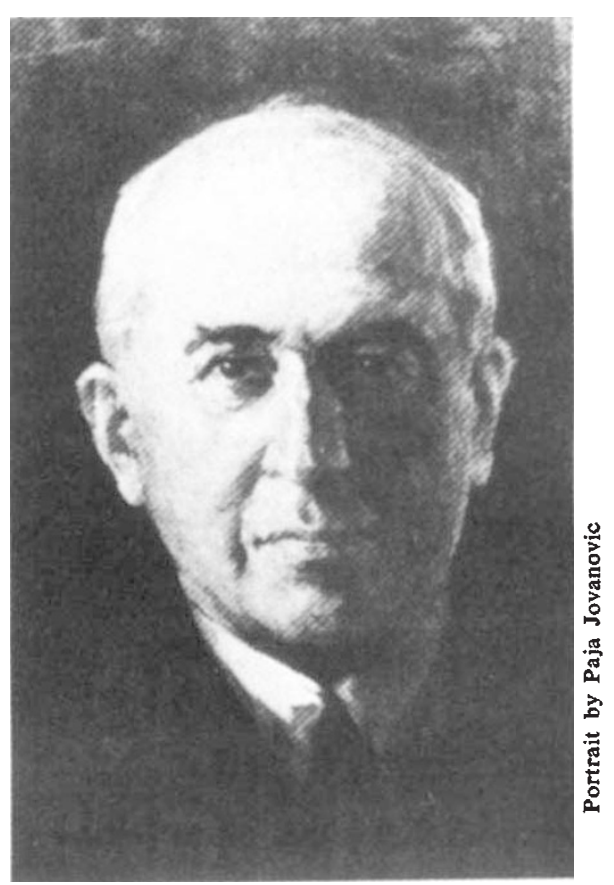

molecular biology. It allows the reader for a few hours to participate in the intrigues, the mystery, and the excitement of scientific discovery. I heartily recommend this book not only to my fellow Earth scientists but also to their wives, children, neighbours and acquaintances who find it difficult to comprehend our preoccupation with the past.

W. S. Broecker is Newberry Professor of Geology at Columbia University, and Associate Director of the Lamont-Doherty Geological Observatory, Palisades, New York.

\section{Scientific journalese}

\section{Joe Schwartz}

Experiencing Science. By Jeremy Bernstein. Pp. 275. (Basic Books: New York; Burnett Books/André Deutsch: London, 1978.) $£ 5.95$.

JEREMY BERNSTEIN could be called the C. P. Snow of the 1960 s. Writing with $I$ an authority and confidence characteristic of Snow's novels Bernstein communicated a literate insiders view of o the high energy physics community during the easy money days of ten years ago. The present collection of articles, published from 1969 to 1978 reflects the strains induced in science by the financial stringencies of the seventies a and a different social and political cli- mate. Bernstein begins to show signs of struggling with the changed circumstances, but the results are not as satisfying as his earlier work.

His evaluation of Nora Sayre's book on Rosalind Franklin suffers from an insensitivity to the very real difficulties women experience in a male dominated profession. By concentrating on the facts of the case as presented in Robert Olby's book, The Path to the Double Helix, Bernstein chooses to avoid the underlying issues of patriarchy and male domination that prompted Ms Sayre to write her book in the first place. This attitude intervenes to weaken an interesting attempt to describe Bernstein's personal struggle to understand Goedel's Theorem (there exist true mathematical statements that are unprovable). Bernstein introduces a lady friend, K., with whom he will discuss his difficulties. Unfortunately all she does is cook him a meal, listen to a lecture and go to bed. She has no lines of her own. K. 